\title{
Patient safety and error management in endoscopy ATR Axon MD FRCP
}

\author{
Anthony Thomas Roger Axon \\ Department of Gastroenterology, University of Leeds, England, UK
}

\begin{abstract}
\end{abstract}
\section{Introduction}

Flexible digestive endoscopy first became widely used in the late 1960s. At that time when complications were encountered, endoscopists learned from the mistakes they made, but then procedures were mainly diagnostic in nature and by today's standards relatively few examinations were performed, so the potential risk of complications was limited.

In the $21^{\text {st }}$ century, endoscopy has become the gastroenterologists' major clinical activity, it is indicated for most of our diagnostic and preventative work and increasingly for therapy. The large number of endoscopies and the expanding proportion of interventional procedures today mean that at present there is a greater risk of endoscopy-related complications. Our first duty to patients is to do no harm and for this reason, national and international endoscopy societies have, in recent years, focused on trying to improve

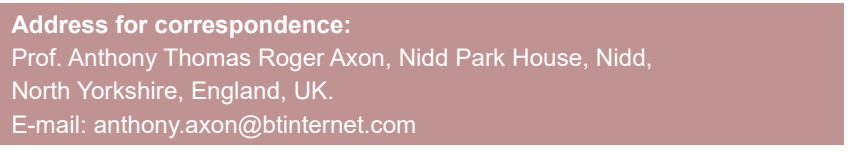

\begin{tabular}{|l|c|}
\hline \multicolumn{2}{|c|}{ Access this article online } \\
\hline \multirow{2}{*}{$\begin{array}{l}\text { Website: } \\
\text { www.jdeonline.in }\end{array}$} & Quick Response Code \\
\hline DOI: & \\
10.4103/0976-5042.193734 & \\
\hline
\end{tabular}

the quality of the endoscopic service that we provide, ${ }^{[1-5]}$ the most important aspect of which is patient safety.

The expansion, increasing complexity, and cost of endoscopy have meant that the provision of an efficient endoscopy service has become the responsibility of hospital management rather than that of the doctors. This change in governance has been both necessary and desirable because if properly exercised and if well-designed protocols are followed, it promotes efficiency and improves patient safety. This change, however, has led to a reduction in the doctor/patient interface, so it is essential that the profession should maintain a close surveillance over the way the endoscopy unit is organized and in particular that quality assurance is employed in endoscopy services to ensure that appropriate protocols are adopted, training is monitored, and outcomes are recorded and are regularly reviewed by a medically qualified team.

\section{Why do Errors Arise?}

Problems do arise during endoscopy [Table 1], some are unavoidable but most can be prevented or the risk can be

This is an open access article distributed under the terms of the Creative Commons Attribution-NonCommercial-ShareAlike 3.0 License, which allows others to remix, tweak, and build upon the work non-commercially, as long as the author is credited and the new creations are licensed under the identical terms.

For reprints contact: reprints@medknow.com

How to cite this article: Axon AT. Patient safety and error management in endoscopy ATR Axon MD FRCP. J Dig Endosc 2016;7:93-7. 
minimized by taking appropriate precautions [Table 2]. These steps include ensuring that the endoscopy suite is well planned, the equipment is effective and well maintained, there is adequate training and supervision of new endoscopists and other professional and support staff, well-prepared in-house protocols have been agreed and are carefully followed, and outcomes are audited and the findings are acted upon.

\section{How to Reduce the Risk of Complications}

\section{Teamwork}

Teamwork is essential for an effective, efficient, and safe endoscopy service. Teams vary in size and make up according to the number and variety of procedures that are performed in the particular endoscopy unit, but it will include endoscopists, nurses, health-care assistants, reception and secretarial staff, and others who are responsible for cleaning, patient in-hospital transport, anesthetics, radiological safety, and liaison. The leaders of the team will usually be an endoscopy director and a chief nurse. An endoscopy users' committee drawn from the team should meet regularly and must be responsible for the policy and strategy of the unit. This will include safety,

\begin{tabular}{l}
\hline Table 1: Many things can go wrong before, during, and after \\
endoscopy \\
\hline Broken tooth \\
Allergic reactions \\
Cross-infection \\
Hypotension \\
Apnea \\
Inhalation \\
Hypoglycemia \\
Wrong procedure \\
Cardiac arrest \\
Wrong injection \\
Perforation \\
Bleeding \\
Splenic rupture \\
Pancreatitis \\
Cholangitis \\
PEG MRSA \\
Glutaraldehyde colitis \\
Specimen mix-up
\end{tabular}

PEG=Percutaneous endoscopic gastrostomy, MRSA=Methicillin-resistant Staphylococcus aureus

\begin{tabular}{l}
\hline Table 2: Prevention is better than cure \\
\hline Endoscopy suite design \\
Reprocessing of endoscopic equipment \\
Patient pre-assessment \\
Team pause \\
Sedation and monitoring \\
Life support training and equipment \\
Labeling, cleanliness, and drug administration \\
Post-procedure care \\
Discharge information
\end{tabular}

training, the preparation of protocols, clinical auditing, and strategy for the future. It should also include a representative of the hospital management.

\section{In-house protocols}

In the early days, there were few purpose-designed endoscopy suites or recovery areas and no nurses were specifically trained to assist at endoscopy. The risk of cross-infection was ignored because it was thought that provided an instrument was socially clean and did not break a mucosal barrier, no infective danger could result.

The importance of careful pre-procedure reprocessing became recognized following the publication of outbreaks of endoscopy-induced gastroenteritis and serious (often lethal) Pseudomonas cholangitis arising from endoscopic retrograde cholangio-pancreatography (ERCP). The vital importance of carefully audited reprocessing and tracking of equipment by properly trained staff remains a critical aspect of safety in endoscopy. Recent experiences of biliary sepsis confirm the importance of monitoring endoscopy outcomes today, in particular, when using the newly designed equipment. ${ }^{[6]}$

In-house clinical protocols should be designed and approved by the endoscopy users' committee. Excellent national and international protocols are available relating to instrument reprocessing, antibiotic prophylaxis, management of gastrointestinal bleeding, endoscopy in people with diabetes, endoscopy in anticoagulated patients, percutaneous endoscopic gastrostomy placement, and colonoscopic surveillance for polyps and for inflammatory bowel disease. These protocols are valuable for reference. However, they are long and complicated and are not specifically designed for day-to-day use. They include a considerable amount of evidence-based data and discussion and may be difficult to access immediately, may overlook geographical differences and the availability of equipment, and eventually go out of date. They should be used as guides for the development of user-friendly didactic in-house protocols that are based on official guidelines but should focus on simplified easy-to-follow instructions available for immediate access in hard copy or on computers in the endoscopy rooms. They should be prepared by the endoscopy staff themselves (a useful learning exercise) and must be reviewed regularly. In-house protocols should extend to all activities in the endoscopy room. Potential risks to patient safely include the injection of the wrong drug and inaccurate labeling of specimens [Table 3].

\section{Patient preparation before the examination}

Certain risks to patient safety can be avoided by ensuring that the planned examination is for an appropriate indication. Patients must be fully informed about the details of their examination well before the date of the procedure. They should receive a written explanation as to what to expect during 
their examination so that they can inform the team about any comorbidities or health conditions. These include diabetes, allergies, ischemic heart disease and respiratory conditions, prescribed drugs (such as anticoagulants), and pregnancy. The written information leaflet is not however a substitute for a careful pre-procedure interview and examination on the day of the procedure by a trained nurse. This must take place to check on general health and fitness and to ensure whether the patient has been properly prepared, has fasted, is fit for sedation (if it is to be used), and that he/she has someone to accompany him/her home after the procedure [Table 4].

A recent innovation that is recommended following admission to the procedure room before starting the examination is the "team pause." The principal reason for this is to check that he/she is the correct patient, that the procedure is the one requested, and to see whether there has been any recent clinical, pathological, or radiological data that might impact on the examination.

\section{During the examination}

If sedation is used, the patient is at a greater risk of respiratory and cardiovascular complications, so appropriate monitoring must be undertaken [Table 5]. If in addition the patient is at a greater risk because of comorbidity, it may be appropriate to seek anesthetic assistance [Table 6].

All clinical endoscopic staff should have undergone regular periodic basic life support training. A person trained in advanced life support should be rapidly available and mobile cardiac arrest facilities must be kept in close proximity to the endoscopy suite.

\section{After the examination}

Post-procedure care is required because endoscopic complications may not present until after the patient has reached the recovery room or has returned home. It is especially important that the patient or accompanying friend or relative is given a contact telephone number to call in the event of an unexpected clinical problem. The patient should not be discharged home until the staff are happy that the patient is fit to go home [Table 7].

\section{Error Management}

\section{Immediate management}

When a complication does arise, it has to be dealt swiftly and effectively. The availability of clips has enabled many cases of bleeding and perforation previously referred for surgery to be managed endoscopically and so it is important for endoscopists to gain expertise in the use of this equipment during their training.

In the event of a complication, the patient must be given a full and truthful explanation as to its nature and why it happened and the clinician should express his or her concern. If the complication has been caused by a clinical error, an apology should be given. If serious, the primary endoscopist will usually remain engaged in the subsequent management, but sometimes if it is thought to be desirable, a transfer of care to a colleague or surgeon will add depth and objectivity to the team, allowing them to make difficult judgment decisions more

\begin{tabular}{l}
\hline Table 3: Miscellaneous causes of risk to patients \\
\hline Handling of sharps \\
Fire safety \\
Storage of supplies \\
Inadequate cleaning of surfaces \\
Failure of hand washing and hygiene \\
Labeling of specimens \\
IV injection protocol (wrong drug injected) \\
IV=Intravenous \\
\hline Table 4: Preassessment \\
\hline Queries relating to the information sheet provided when booking \\
Anticoagulants and antiplatelet drugs \\
Comorbidities (e.g., diabetes) and allergies \\
Pregnancy \\
Substance abuse \\
Previous problems with sedation \\
BP, pulse, and oxygen saturation \\
ASA status
\end{tabular}

$\mathrm{BP}=$ Blood pressure, $\mathrm{ASA}=$ American Society of Anesthesiologists

\section{Table 5: Sedation monitoring}

Monitor BP, pulse, oxygen saturation, maybe ECG

Give supplemental oxygen

Two assistants (one for patient, one for procedure)

Tipping trolley

Suction, oxygen, and bag mask available

All staff trained in basic life support

Someone trained in advanced life support available

Resuscitation trolley available and checked

$\mathrm{BP}=$ Blood pressure, $\mathrm{ECG}=$ Electrocardiogram

Table 6: Is anesthetist assistance required?

Strong consideration

ASA Status IV and V

History of alcohol or substance abuse

Pregnancy, morbid obesity, neurologic or neuromuscular disorders

Patients who are uncooperative or delirious

May be required

ERCP

Stent placement in the upper gastrointestinal tract

Endoscopic ultrasound

Complex therapeutic procedures

$\mathrm{ERCP}=$ Endoscopic retrograde cholangio-pancreatography

Table 7: Postprocedural assessment

Stable vital signs for at least 1 hour

Alert and oriented to time, place, and person

No excessive pain, bleeding, or nausea

Able to dress and walk with assistance 
objectively. ${ }^{[7]}$ If this is done, the patient must understand who is now responsible for his/her clinical care.

\section{The legal position}

The patient may seek redress through the courts for an injury if it is thought that it has arisen as a result of negligent management. Unless the doctor has acted in a reckless manner, it is dealt by civil law not by criminal law in most jurisdictions. This means that if the case is settled in favor of the claimant, the doctor or health-care provider has to pay financial compensation as directed by the court but individuals are not at risk of a prison sentence.

To succeed in a claim, the claimant has first to convince the court that a breach of duty has taken place and that no reputable body of practitioners would have condoned it. That is to say, the clinical decision-making and/or the performance of the procedure would not have been supported by any reputable body of medical professionals skilled in that particular field. Second, the claimant must show that on a balance of probabilities (more likely than not) the breach of duty had caused or contributed to the injury, loss or damage claimed, and that the patient would not have suffered that injury but for the breach of duty. Only if both of these requirements are met, will the defendant be liable to pay financial compensation. ${ }^{[8-10]}$

\section{Reducing the Risk of Litigation}

\section{Patient-doctor relationship}

Following a complication, patients and their relatives will be concerned, frightened, and often angry. They may immediately decide that they will make a complaint and consider litigation whether or not the complication was caused by chance or has arisen because of clinical error. It is essential therefore at an early stage for the clinician to provide a clear explanation as to why the complication arose, what will be done to rectify it, and to reassure the patient and family that everything possible will be done. This approach helps to restore trust and reduce anger. Patients are reluctant to sue a doctor if they have established a good relationship with him or her and if they feel that he or she has done their best; "Good-nature and good-sense must ever join; To err is human, to forgive, divine."[11]

\section{Informed consent}

Many endoscopic complications arise by chance and are not the fault of the clinical staff, equipment used, or the system of management. ${ }^{[12]}$ However, even these may lead to a complaint and potential litigation if the patient had not been made aware of the possibility of that complication before agreeing to undergo the endoscopy. It is essential therefore that patients understand the nature of any procedure that is planned, why it is being done, what potential risks are involved, the likelihood of the risk, and what alternative clinical options are available other than endoscopy. Patients have the right to decide whether to undergo a procedure or not, ${ }^{[13,14]}$ and if they have not given their informed consent and a complication does arise, under the UK law, the court is likely to find in their favor.

Informed consent, therefore, empowers the patient, enabling him/her to determine whether or not to undergo the intervention recommended. However, informed consent is also a protection for the endoscopist in the event of a chance complication. For these reasons, the patient should be provided with a written explanation setting out the possible complications before the examination and there must be an opportunity for the patient to bring any concern or query to the staff before the consent form is signed.

\section{Guidelines, standard practice, and notes}

Litigation may not be started until months or even years after a complication has occurred and by then it may be difficult to recall the details as to what or why a particular line of action was taken, so legible, detailed notes must be made.

Guidelines and standard practice should be followed, but there will be occasions where recommendations are controversial or there may be clinical reasons why a different course of action should be taken. It is particularly important therefore to record why a clinical decision was made in the event that it deviated from accepted guidelines or from usual practice.

\section{Prevention of future errors}

Mistakes can arise in any unit and if they do, they should not be ignored or covered up even if no harm resulted. They should be investigated to discover whether it was caused by a failure of clinical staff, equipment, system, by chance or a combination of these. Unless a cause of failure can be identified, it cannot be rectified and it is likely to happen again. For these reasons, it is essential that patient outcomes are recorded and are regularly audited. For example, if there is a sudden increase in post-endoscopic colitis symptoms, this may draw attention to inadequate rinsing of a colonoscope causing glutaraldehyde-associated colitis, or a sudden increase in post-ERCP pancreatitis may be attributable to an over-enthusiatic endoscopist who is injecting the pancreatic duct repeatedly while trying to enter the bile duct.

\section{Conclusion}

It is the most distressing experience in a professional life for a doctor to harm a patient as a consequence of a clinical error. Unfortunately, this will occasionally happen in the best centers. The risk of error can be reduced by good team management, by paying attention to preprocedural consent and health status, by adherence to protocols, by careful monitoring during and after the procedure, and by appropriate advice on discharge. Regular clinical audit helps to identify potential future risks.

Error is easier to manage if a good doctor-patient relationship has been established before it arises. In the event that a 
complication does occur, it should be dealt rapidly and effectively, its cause must be explained honestly with reassurance that everything possible will be done.

Clear, detailed, legible notes are helpful if a case comes to litigation.

\section{Financial support and sponsorship}

Nil.

\section{Conflicts of interest}

There are no conflicts of interest.

\section{References}

1. Bjorkman DJ, Popp JW Jr. Measuring the quality of endoscopy. Gastrointest Endosc 2006;63 4 Suppl:S1-2.

2. Report of a joint ASGE/ACG Taskforce on Quality in Endoscopy. Gastrointest Endosc 2006;63:S3-38.

3. Rembacken B, Hassan C, Riemann JF, Chilton A, Rutter M, Dumonceau JM, et al. Quality in screening colonoscopy: Position statement of the European Society of Gastrointestinal Endoscopy (ESGE). Endoscopy 2012;44:957-68.

4. Faigel DO, Cotton PB; World Organization of Digestive Endoscopy. The London OMED position statement for credentialing and quality assurance in digestive endoscopy. Endoscopy 2009;41:1069-74.

5. British Society of Gastroenterology Endoscopy: Clinical Guidance.
Available from: http://www.bsg.org.uk/clinical-guidance/endoscopy/ index.html. [Last accessed on 2016 Jul 18].

6. Ross AS. Enhanced methods for duodenoscope reprocessing: Answers or just more questions. Gastrointest Endosc 2016;84:263-5.

7. Richter JM, Kelsey PB, Campbell EJ. Adverse event and complication management in gastrointestinal endoscopy. Am J Gastroenterol 2016;111:348-52.

8. Medical Protection Society Professional Support and Expert Advice Clinical Negligence Claims - What to Expect. Available from: http:// www.medicalprotection.org/uk/resources/factsheets/england/ england-factsheets/uk-eng-clinical-negligence-claims-what-to-expect. [Last accessed on 2016 Jul 18].

9. Agarwal AK. Medical Negligence: Law and Interpretation. Indian Institute of Management; 2011. Available from: http://www.iimahd.ernet. in/assets/snippets/workingpaperpdf/12616804582011-03-03.pdf. [Last accessed on 2016 Jul 18].

10. Murthy KK. Medical negligence and the law. Indian J Med Ethics 2007;IV:116-7.

11. Pope A. An Essay on Criticism Part II, 1711. Available from: http://www. rpo.library.utoronto.ca/poems/essay-criticism-part-212. [Last accessed on 2016 Jul 18].

12. Sonnenberg A. Adverse outcomes: Why bad things happen to good people. Clin Gastroenterol Hepatol 2015;13:820-3.e1

13. Nandimath OV. Consent and medical treatment: The legal paradigm in India. Indian J Urol 2009;25:343-7.

14. World Health Organization. A Declaration on the Promotion of Patients' Rights in Europe; European Consultation on the Rights of Patients Amsterdam; 28-30 March, 1994. Available from: http://www. who.int/genomics/public/eu_declaration1994.pdf. [Last accessed on 2016 Jul 18]. 